\title{
Safe Extraforaminal Docking and Floating Technique in Transforaminal Endoscopic Discectomy for Thoracolumbar Junction for Calcified Disc Herniation: A Case Report and Technical Review Junction for Calcified Disc Herniation
}

\author{
Pang Hung $\mathrm{Wu}^{1,2}$, Hyeun Sung Kim${ }^{1}$, Harshavardhan D Raorane ${ }^{1}$, Dong Hwa Heo ${ }^{1}$, Yeon Jin $\mathrm{Yi}^{1}$, \\ Kyung-Hoon Yang ${ }^{1}$, Il-Tae Jang ${ }^{1}$ \\ ${ }^{1}$ Department of Neurosurgery, Nanoori Hospital Gangnam, Seoul, Korea, ${ }^{2}$ National University Health Systems, Juronghealth Campus, Singapore
}

Corresponding Author: Hyeun-Sung Kim, MD, PhD Department of Neurosurgery, Nanoori Hospital Gangnam, 731, Eonju-ro, Gangnam-gu, Seoul 06048, Korea

Tel: $+82-2-6003-9767$

Fax: +82-2-3445-9755

E-mail: neurospinekim@gmail.com

*Both Dr. Pang Hung Wu and Dr. Hyeun Sung Kim contributed equally as first author of this publication.

Received: October 17, 2019 Revised: March 25, 2020 Accepted: March 27, 2020

\begin{abstract}
Objective: Thoracolumbar disc herniation is an uncommon disabling spine condition. Methods: There is relatively large dura expansion to lateral recess in the thoracolumbar junction leading to potential hazard in docking and execution of endoscopic transforaminal discectomy. Mobile outside in technique had been executed successfully in lumbar disc herniation, we modified the technique to allow safe docking and execution of discectomy in thoracolumbar junction. Herein, we report a case of thoracic twelve lumbar one disc paracentral disc herniation treated with floating transforaminal endoscopic discectomy under local anesthesia. Results: A 46-year-old man with a 3-months of severe back and radicular pain on the right groin. A diagnosis of right T12/L1 paracentral disc herniation was made, based on clinical and radiological investigations. He underwent percutaneous transforaminal endoscopic thoracolumbar discectomy with floating technique. Over a follow-up of 6 months, a favorable outcome was noted. Conclusion: Floating technique of transforaminal endoscopic thoracolumbar junction discectomy is a safe and an effective surgical approach for the treatment of thoracolumbar disc herniation. In patients with thoracolumbar disc herniation, careful patient selection and evaluation neural elements anatomy in relation to bony anatomy is important in preparation for endoscopic decompression.
\end{abstract}

Key Words: Thoracolumbar junction, Calcified disc herniation, Transforaminal, Endoscopic discectomy, Floating technique

\section{INTRODUCTION}

Thoracic disc herniation is a rare condition occurring mainly in between T8-L1. Although a rare condition, patients typically presents with significant morbidity, such as intractable back or radicular pain, neurological deficits, scoliotic posture and myelopathy signs ${ }^{6,20}$. Calcified disc herniation is a challenging surgical scenario especially giant calcified disc herniation which occupied more than 40\% spinal canal occupation presenting with myelopathy. Calcified disc tends to have dura extension with a higher risk of dura tear and higher post -operative complications ${ }^{6)}$. Smaller disc had been described to be remove through various endoscopic approaches which is as effective as open surgery with potential less muscle stripping and post-operative pain ${ }^{1,16)}$.
Several authors described various transforaminal approach through docking on Kambin's triangle and foraminoplasty ${ }^{218}$. Dura is more expanded in the thoracolumbar junction with a higher dura sac ratio as we move more proximally from lumbosacral region to thoracolumbar junction ${ }^{15}$. The authors would like to use this case example to highlight the importance of extraforaminal docking and introduce a floating technique to perform discectomy safely in the region of thoracolumbar spine.

\section{MATERIALS AND METHOD}

\section{History}

A 46 years old man was admitted to our department with 
3 months history of severe pain of Visual Analog Scale (VAS) 9 with radicular pain over the right groin. He had no weakness and no bladder or bowel dysfunction. He had undergone 3 months of conservative treatment with no improvement. Physical examination showed he had significant back spasm and mild numbness over the right medial groin but otherwise power and reflexes were normal. Magnetic resonance imaging showed right paracentral disc herniation of T12/L1 disc. There was low downward migration of the disc (Fig. 1(A) and (B)). Computer Tomography (CT) Scan showed there was a calcified disc at the concordant region of right paracentral T12/L1 disc space. A clinical diagnosis of right paracentral T12/L1 calcified prolapsed intervertebral disc was made. Surgical decompression options were discussed, the patient was scheduled to undergo right percutaneous endoscopic transforaminal decompression of T12/L1.

\section{Surgical Treatment}

Percutaneous uniportal transforaminal endoscopic thora columbar decompression was performed at the T12/L1 level under local anesthesia. The patient was positioned in the prone position on a radiolucent table with padding over the axilla, waist and bony prominences. Intraoperative C-arm fluoroscopy was utilized to localize and plan the puncture route. The entry point and trajectory was planned preoperatively using MRI and CT scan to be docked on extraforaminal disc region lateral to right L1 pedicle (Fig. 1(A), (B) and (C). In this case the incision was made $5 \mathrm{~cm}$ off the mid spinous process and in line with T12/L1 disc space. After the subcutaneous tissue and trajectory were infiltrated with $5 \mathrm{~mL}$ of $1 \%$ lidocaine through a $23 \mathrm{G}$ needle. We checked for the free epidural space by injecting $1.5 \mathrm{~mL}$ of contrast dye under the image intensifier (Fig. 2(B) and (C)). We saw a diffuse spread of dye in epidural space rather than a localised dye to one nerve root. Once it was confirmed safe to dock by epidurogram with contrast, we provided local anesthesia by injecting $8-10 \mathrm{~mL}$ of $1 \%$ lidocaine in the foraminal space followed by a booster injection of $2-4 \mathrm{~mL}$ of $1.6 \%$ lidocaine ( $8 \mathrm{~mL}$ of $2 \%$ lidocaine with $2 \mathrm{~mL}$ of saline plus $0.05 \mathrm{~mL}$ of epinephrine) 4-5 min after the initial injection and docked the working channel through obturator on extraforaminal disc as planned. (Fig 2(D) and 2(E)). After docking, we introduced the endoscope into the extraforaminal space. We used the beveled type working cannula with an $8 \mathrm{~mm}$ outer diameter. The endoscope had a $30^{\circ}$ viewing angle, a $7.3 \mathrm{~mm}$ outer diameter, a $4.7 \mathrm{~mm}$-diameter working channel, and $251 \mathrm{~mm}$ of total length. The entire procedure was performed using constant saline irrigation. We performed soft tissue dissection and clearly identified the pars, transverse process of T12 and facet joint of T12/L1 (Fig. 3(A)).
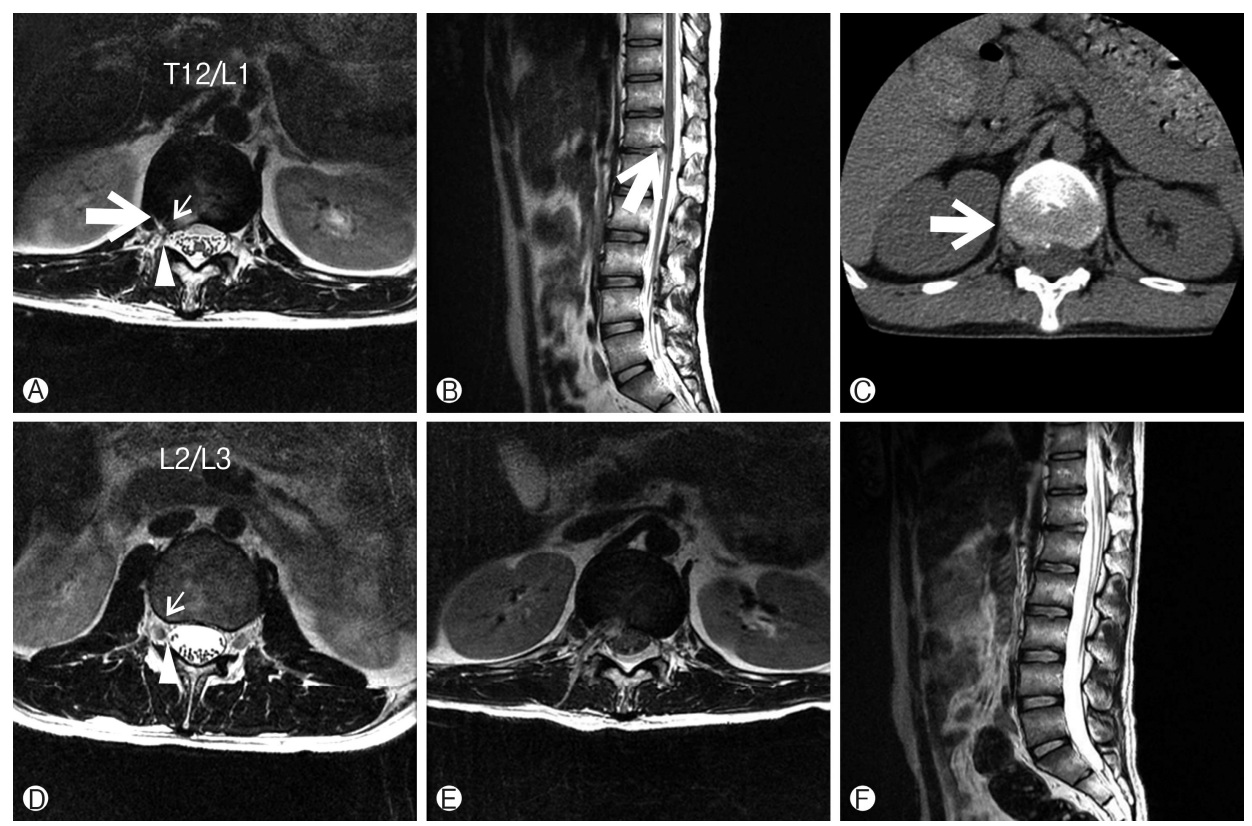

Fig. 1. A Paracentral herniation of T12/L1 disc with annular tear (small arrow), lateral edge of dura is shown lateral to the usual docking area of Kambin triangle (broad arrow head), the proposed docking point of the working channel (big arrow). (B) Sagittal view of T12/L1 paracentral disc herniation with mild downward migration of the disc. (C) CT scan showing calcification of the T12/L1 paracentral disc herniation (large arrow shows corresponding proposed docking point of extraforaminal disc region). (D) L2/3 Disc space showing lateral edge of dura (broad arrow head) is close to but medial to the docking point of Kambin triangle. (E) and (F) Post-operative day $1 \mathrm{MRI}$ showing discectomy of the herniated disc at T12/L1 paracentral region. 

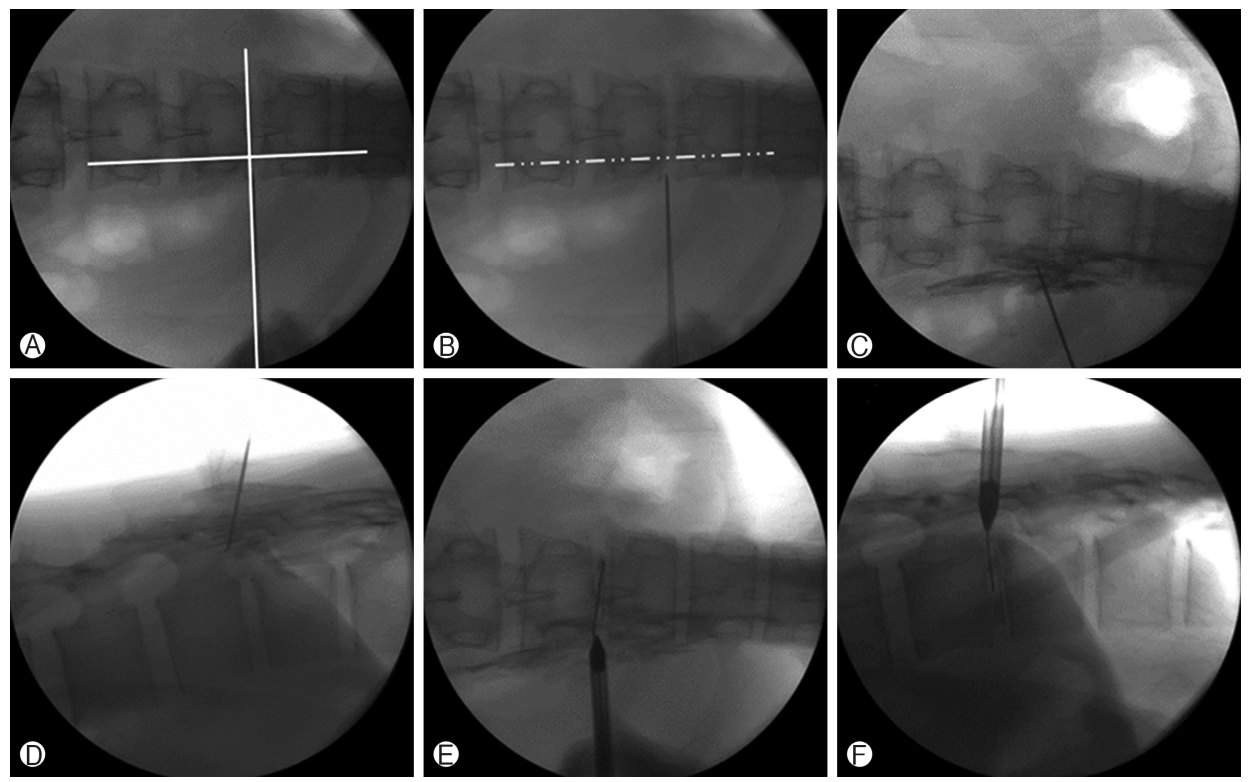

Fig. 2. A Kambin Triangle docking with intersection of medial pedicular line and disc space of T12/L1. B AP view of docking lateral to lateral pedicular line lateral to intended Kambin Triangle. (C) and (D) epidurogram under fluoroscopy with contrast showing diffuse flow of the contrast which suggests docking is lateral to dura. (E) and $(F) A P$ and lateral view showing the docking of extraforaminal disc region after indigo carmine discography.
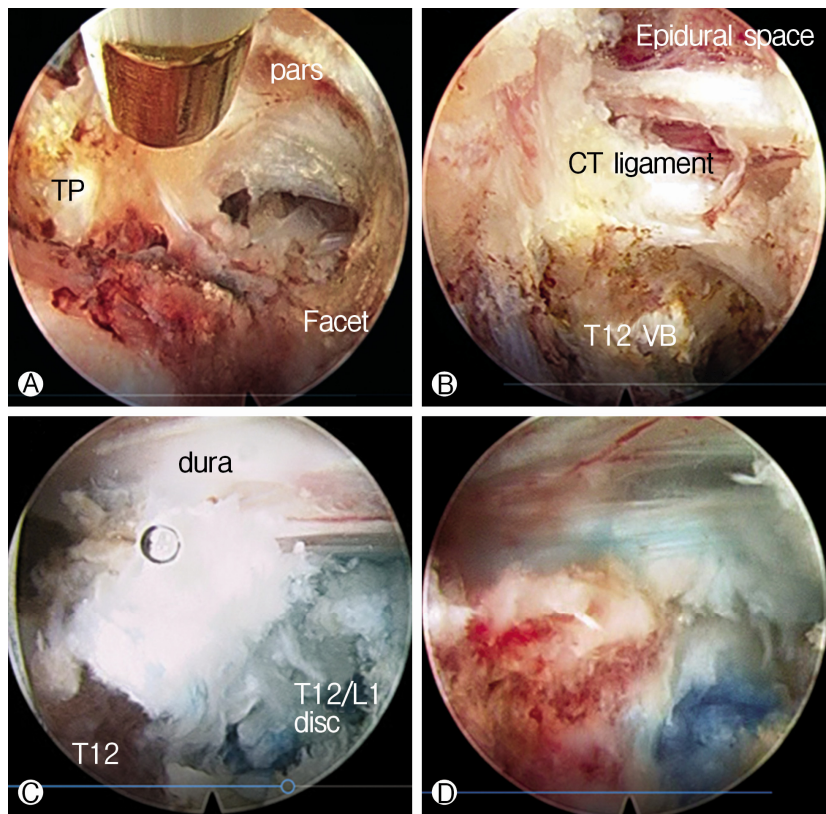

Fig. 3. (A) Soft tissue dissection to expose the pars, transverse process and facet of T12. (B) After partial bony resection of transverse process, pars and facet joint and soft tissue dissection of costotransverse ligament, posterior inferior edge of T12, partially resected costotransverse ligament and epidural space is seen. (C) T12/L disc exposed after further bony and soft tissue dissection. (D) Half and half view with posterior longitudinal ligament separating the 2 halves, showing free pulsating and floating dura under irrigation fluid and bony resected superior inferior edge of $\mathrm{T} 12$ and discectomy site.
Intertransverse and costotransverse ligaments were taken down, slight amount of bony drilling of the transverse process and pars of T12 to provide the correct trajectory for the disc fragment. We then proceed to drill slight amount of inferior articular process of T12 and superior articular process of L1 to allow a more horizontal angle of access to expose the epidural space and disc of T12/L1 (Fig. 3B). We drill the posteroinferior corner of T12 vertebra to further expose T12/L1 disc. The authors felt that it was necessary to perform peridiscal bony dissection to ensure adequate space for drilling and dissection of calcified disc without maneuvers of the spinal cord. We decompressed the neural element and disc space by doing annulotomy and discectomy at this point of time. At the region of calcified disc, we use a combination of drilling and pituitary rongeur to perform the discectomy while carefully preserving the posterior longitudinal ligament. After adequate decompression and discectomy, we checked the half and half view with dura well decompressed and pulsating separated by an intact posterior longitudinal ligament and a decompressed T12/L1 disc space. Free floating dura with visible pulsation in the irrigation fluid was seen as adequate decompression (Fig. 3(D)). We inserted a drain under direct endoscopic vision and withdrew the instruments with sutures on closure. Post-operative day one MRI showed decompressed T12/L1 lateral recess (Fig 1(C) and (D).

\section{Post-operative Outcome}

Patient had improved symptomatically to VAS 0 . He started ambulating on the evening of operation and scheduled for physi- 
otherapy on discharge on post-operative day 2 and had remained asymptomatic at 6 months follow up when he was discharged.

\section{DISCUSSION}

Thoracic disc herniation (TDH) is a disabling condition that is technically difficult to manage. Symptomatic thoracic disc herniations are rare conditions with an incidence of 1 in 1 million patients per year ${ }^{3)}$. Most of the thoracic disc herniation occurs in the lower third of the thoracic spine and majority are subclinical $^{5}$. Intervertebral disc calcification is a common incidental finding in chest and abdominal radiographs with a prevalence of 5 to $6 \% \%^{19)}$. Calcification of the disc is more common in elderly population with chronic disc herniation ${ }^{13)}$. Combination of calcified thoracic disc herniation in the young is rare as presented in this patient. Due to frequently existing calcification, CT scan should be ordered to assess the consistency of the disc to plan the bony dissection required in surgery as well as surgical approaches.

TDH discectomy and decompression is a challenging area of anatomy and there are many approaches to thoracic disc herniation. One common theme is that surgical manipulation of thoracic spinal cord is not advisable. Open approaches such as transpedicular ${ }^{14)}$, costotransversectomy ${ }^{11)}$ and lateral extracavitary ${ }^{10)}$ all of which are associated with significant morbidity with significant postoperative complications which include spinal cord injury, dura tear, pulmonary infection, epidural hematoma, and infection of the incisional wound. Despite its significant perioperative comorbidity, open approaches provide limited access to the thoracic disc ${ }^{8}$. In order for good open approach to thoracic disc, there is large amount of bony resection with a possibility of instability and required fusion procedure. Each of the open approaches has its main advantages and disadvantages but outcomes of minimally invasive surgery seems to have a favorable trend ${ }^{17)}$.

There is a paucity of literature on endoscopic thoracic approaches due to limited number of cases. They are mainly described as interlaminar ${ }^{7,12)}$ and transforaminal approaches ${ }^{2,4,18)}$. One common theme in the literature is docking at the Kambin triangle with foraminoplasty under the guidance of fluoroscopy ${ }^{2,18)}$. The authors felt that due to the likely presence of dura sac at or even lateral to the Kambin triangle in the thoracolumbar region and even upper lumbar region, fluoroscopic guided docking on Kambin triangle within the foraminal region is fraught with possible danger ${ }^{15)}$ (Fig. 1(A) and 1(D).

The authors proposed that docking for uniportal endoscopic thoracic decompression should be on extraforaminal disc region, lateral to pedicle line. Intraoperative epidurogram is essential to outline safe trajectory of the needling and guidewire. Alarm should be raised if epidurogram highlight a particular nerve rather than diffuse appearance. As thoracic twelve and lumbar one nerve root does not radiate to the lower limb, reliance of radicular pain on docking under conscious sedation can give a false sense of security. Intraoperative discogram with mixed contrast and indigo carmine is beneficial to guide us on the amount of discectomy to be done and the location of the disc (Fig. 2).

The mobile outside in technique had been performed with good outcomes in lumbar spine ${ }^{9}$. With that background experience, the authors proposed to dissect tissue under direct endoscopic vision, using a similar floating endoscope technique to maneuver the endoscope with clear view after soft tissue dissection on the pars, tranverse process and facet joint in the initial phase of surgery. Dissection of costotransverse and intertransverse ligaments were necessary to gain direct visualization of the target disc space and its associated bony obstruction. For calcified disc like this case, one should have a low threshold of partial bony resection of the posterior inferior corner of the cephalad segment of the vertebra body adjacent to disc space; in this case, the T12 verterbra body (Fig. 3). Disectomy should be thorough, the presence of indigo carmine in acidic prolapsed disc and clear half and half view of dura after decompression provides good visual analysis of adequate decompression.

To the best of our knowledge, this is the first report of extraforaminal disc docking and floating endoscopic technique to decompress thoracolumbar junction calcified herniated disc. With high clinical suspicion of herniation of disc through a thorough clinicoradiological evaluation establishing the feasibility of endoscopic decompression and the potential difficulty in mobilizing the disc. Further attention should be paid on the lateral margin of the dura in relation to pedicle and facet joint with a high likelihood of encroachment of dura to the "safe" Kambin's triangle corridor in thoracolumbar and high lumbar spine which precludes the "blind" fluoroscopic guided docking and foraminoplasty on Kambin's triangle. With careful evaluation and execution of this technique, the authors hope to minimize the complications associated with the dreaded thoracolumbar disc herniation surgery.

\section{CONCLUSION}

With careful preoperative planning using both $\mathrm{CT}$ and MRI scan, an extraforaminal disc docking and subsequent floating endoscopic technique allows safe transforaminal endoscopic discectomy of paracentral calcified disc in thoracolumbar junction. This technique can be considered when surgeon decides to employ endoscopy as a surgical treatment method to perform discectomy for T12/L1 paracentral disc herniation.

\section{REFERENCES}

1. Ahn Y: Endoscopic spine discectomy: indications and outcomes. International Orthopaedics 43:909-916, 2019

2. Bae J, Chachan S, Shin S-H, Lee S-H: Percutaneous endoscopic thoracic discectomy in the upper and midthoracic spine: A technical note. Neurospine 16:148-153, 2019

3. Carson J, Gumpert J, Jefferson A: Diagnosis and treatment of thoracic intervertebral disc protrusions. Journal of Neurology, 
Neurosurgery, and Psychiatry 34:68-77, 1971

4. Choi KY, Eun SS, Lee SH, Lee HY: Percutaneous endoscopic thoracic discectomy; Transforaminal approach. Minim Invasive Neurosurg 53:25-28, 2010

5. Coscia MF, Strate RW: Calcified thoracic disc herniation with paraparesis. A case report. Acta Orthop Scand 64:489-490, 1993

6. Court C, Mansour E, Bouthors C: Thoracic disc herniation: Surgical treatment. Orthopaedics \& Traumatology: Surgery \& Research 104:S31-S40, 2018

7. Hur J-W, Kim J-S, Seung J-H: Full-endoscopic interlaminar discectomy for the treatment of a dorsal migrated thoracic disc herniation: Case report. Medicine 98:e15541-e15541, 2019

8. Hurley ET, Maye AB, Timlin M, Lyons FG: Anterior versus posterior thoracic discectomy: A systematic review. Spine (Phila Pa 1976) 42:E1437-e1445, 2017

9. Kim HS, Adsul N, Kapoor A, Choi SH, Kim JH, Kim KJ, et al: A mobile outside-in technique of transforaminal lumbar endoscopy for lumbar disc herniations. Journal of Visualized Experiments: JoVE, 2018

10. Lau D, Song Y, Guan Z, Sullivan S, La Marca F, Park P: Perioperative characteristics, complications, and outcomes of singlelevel versus multilevel thoracic corpectomies via modified costotransversectomy approach. Spine (Phila Pa 1976) 38: 523-530, 2013

11. Lesoin F, Rousseaux M, Autricque A, Reesaul Y, Villette L, Clarisse J, et al: Thoracic disc herniations: evolution in the approach and indications. Acta Neurochir (Wien) 80:30-34, 1986

12. Miao X, He D, Wu T, Cheng X: Percutaneous endoscopic spine minimally invasive technique for decompression therapy of thoracic myelopathy caused by ossification of the ligamentum flavum. World Neurosurgery 114:8-12, 2018

13. Oitment C, Kwok D, Steyn C: Calcified thoracic disc herniations in the elderly: Revisiting the laminectomy for single level disease. Global Spine Journal 9:527-531, 2019

14. Patterson RH, Jr., Arbit E: A surgical approach through the pedicle to protruded thoracic discs. J Neurosurg 48:768-772, 1978

15. Pierro A, Cilla S, Maselli G, Cucci E, Ciuffreda M, Sallustio G: Sagittal normal limits of lumbosacral spine in a large adult population: A quantitative magnetic resonance imaging analysis. Journal of Clinical Imaging Science 7:35-35, 2017

16. Ruetten S, Hahn P, Oezdemir S, Baraliakos X, Merk H, Godolias G, et al: Full-endoscopic uniportal decompression in disc herniations and stenosis of the thoracic spine using the interlaminar, extraforaminal, or transthoracic retropleural approach. Journal of Neurosurgery: Spine 29:157-168, 2018

17. Sharma SB, Kim JS: A review of minimally invasive surgical techniques for the management of thoracic disc herniations. Neurospine 16:24-33, 2019

18. Telfeian AE, Jasper GP, Oyelese AA, Gokaslan ZL: Technical considerations in transforaminal endoscopic spine surgery at the thoracolumbar junction: report of 3 cases. Neurosurgical Focus 40:E9, 2016

19. Weinberger A, Myers AR: Intervertebral disc calcification in adults: a review. Semin Arthritis Rheum 8:69-75, 1978

20. Zhu Z, Zhao Q, Wang B, Yu Y, Qian B, Ding Y, et al: Scoliotic posture as the initial symptom in adolescents with lumbar disc herniation: its curve pattern and natural history after lumbar discectomy. BMC Musculoskeletal Disorders 12:216-216, 2011 